\title{
Renal Denervation Effects on Myocardial Fibrosis and Ventricular Arrhythmias in Rats with Ischemic Cardiomyopathy
}

\author{
Bin Zhang ${ }^{\mathrm{a}}$ Xiaorong Lia Chu Chen ${ }^{\mathrm{a}} \quad$ Wanying Jiang ${ }^{\mathrm{a}}$ Dasheng Lu ${ }^{\mathrm{a}}$ Qian Liu ${ }^{\mathrm{a}}$ \\ Kai Wang ${ }^{\mathrm{a}}$ Yuhao Yan ${ }^{\mathrm{a}}$ Zhixin Jiang ${ }^{\mathrm{a}}$ Jie Geng ${ }^{\mathrm{a}}$ Hai Xua Qijun Shan ${ }^{\mathrm{a}}$ \\ ${ }^{a}$ Department of Cardiology, the First Affiliated Hospital of Nanjing Medical University, Nanjing, China
}

\section{Key Words}

Renal Denervation • Myocardial Infarction • Ventricular Arrhythmia • Fibrosis • Connexin

\begin{abstract}
Background/Aims: To investigate the impact of renal denervation (RDN) on myocardial fibrosis and ventricular arrhythmias (VAs) in rats with ischemic cardiomyopathy. Methods: An ischemic cardiomyopathy model was reproduced with myocardial infarction (MI) in adult Sprague-Dawley male rats. The RDN/Sham-RDN procedure was performed at 2 weeks after MI. Sham-MI and sham-RDN rats served as the control group. At 4 weeks after RDN, programmed electrical stimulation (PES) was used to induce VAs, including ventricular tachycardia and ventricular fibrillation, in all 3 groups (MI+RDN, MI, and control groups). At the end of PES, heart and kidney samples were harvested. Immunofluorescence labeling was used to investigate the distribution of connexin $43(\mathrm{C} \times 43)$ in the infarcted border zone. Masson's trichrome stain was adopted to determine the degree of cardiac fibrosis. Western blotting was performed to identify the expression of transforming growth factor beta 1 (TGF- $\beta 1$ ), $\alpha$-smooth muscle actin ( $\alpha$-SMA), and Cx43. An enzyme-linked immunosorbent assay (ELISA) was used to detect the serum levels of B-type natriuretic peptide (BNP) and the amino-terminal propeptides of type I and III collagen (PINP and PIIINP, respectively) and the expression level of renal norepinephrine. Results: Compared with the MI group, RDN significantly decreased the inducibility of VAs (MI+RDN $3 / 8$ rats vs. MI 8/9 rats, $\mathrm{P}<0.05$; control $1 / 8$ rats) with PES, reduced myocardial fibrosis estimated by collagen volume fraction (MI+RDN $31.10 \pm 3.97 \%$ vs. MI $54.80 \pm 16.39 \%, P<0.001$; control $4.41 \pm 0.92 \%)$, suppressed TGF- $\beta 1(P<0.01)$ and $\alpha$-SMA $(P<0.001)$ levels, and attenuated both PINP (MI+RDN $41.44 \pm 10.10 \mathrm{ng} / \mathrm{mL}$ vs. MI $95.49 \pm 24.83 \mathrm{ng} / \mathrm{mL}, \mathrm{P}<0.001$; control $11.90 \pm 4.96 \mathrm{ng} / \mathrm{mL}$ ) and PIIINP (MI+RDN $82.12 \pm$ $30.79 \mathrm{ng} / \mathrm{mL}$ vs. MI $124.60 \pm 26.64 \mathrm{ng} / \mathrm{mL}, \mathrm{P}<0.05$; control $64.69 \pm 23.84 \mathrm{ng} / \mathrm{mL}$ ) levels. Moreover, RDN reversed the abnormal myocardial distribution of $\mathrm{C} \times 43$ and its reduction by MI damage $(P<0.01)$. Conclusions: RDN reduced myocardial fibrosis and suppressed VAs in a rat model of ischemic cardiomyopathy.

B. Zhang and X. Li contributed equally to this work.

Qijun Shan, MD, PhD

Department of Cardiology, The First Affiliated Hospital of Nanjing Medical University 300 Guangzhou Road, Nanjing, 210029, Jiangsu Province (China)

Tel. +86 2568136407, Fax +86 (0)25 68303111, E-Mail qjshan@njmu.edu.cn
\end{abstract}

KARGER 


\section{Introduction}

The cause of death varies at different times in ischemic cardiomyopathy after myocardial infarction (MI). Fatal ventricular arrhythmias (VAs) resulting from ischemia lead to sudden death in the acute state of MI. Over time, cardiac remodeling induces VAs leading to death [1]. Hence, any approaches to improve structural remodeling might also have potential antiarrhythmic effects in ischemic cardiomyopathy.

Renal denervation (RDN), which is a novel technique originally used for treating resistant hypertension [2], was shown to exert beneficial effects on multi-organ fibrosis in our previous study [3]. RDN has also been reported to attenuate cardiac remodeling and preserve cardiac function in several studies $[4,5]$. In addition, with respect to VAs after MI, Linz et al. reported that RDN suppressed VAs during acute ischemia events [6], while Nicholas et al. recently demonstrated its role in reducing post-infarction VAs [7]. However, little is known of the mechanisms underlying these phenomena. Thus, the purpose of the present study was to investigate the effects of RDN on myocardial fibrosis and electrophysiology in a rat model of MI-induced ischemic cardiomyopathy and to explore the possible underlying mechanisms.

\section{Materials and Methods}

\section{Animal care}

Male Sprague-Dawley rats (200-220 g) were obtained from Nanjing Medical University Laboratory Animal Center. All experimental procedures were approved by the Ethics Committee of Nanjing Medical University. This study conformed to institutional guidelines for the care and use of experimental animals.

\section{Induction of an ischemic cardiomyopathy model}

An ischemic cardiomyopathy model was generated with MI by ligating the left anterior descending coronary artery, as described previously [8, 9]. In brief, the rats were anesthetized by $2 \%$ pentobarbital sodium $(40 \mathrm{mg} / \mathrm{kg}$ ) before they were endotracheally intubated and mechanically ventilated. The pericardium was opened through a thoracotomy in order to ligate the left anterior descending coronary artery with a $7-0$ silk suture, which was approximately $2 \mathrm{~mm}$ below the tip of the left atrium. After ligation, the surface of the heart turned white immediately, and the thorax was closed. The rats were placed in a $26^{\circ} \mathrm{C}$ environment to help them regain consciousness. The rats were given pethidine ( $40 \mathrm{mg} /$ day) and penicillin $(400,000 \mathrm{U} /$ day) intramuscularly to relieve pain and prevent infection for 3 days. The animals in the control group were subjected to a similar procedure but without coronary artery ligation. All MI procedures were performed by the same operators.

\section{$R D N$}

At 2 weeks after MI, bilateral RDN was conducted in the MI+RDN group, as described previously [10]. Briefly, a vertical back incision was performed under general anesthesia with $2 \%$ pentobarbital sodium ( 40 $\mathrm{mg} / \mathrm{kg}$ ). All of the visible nerves were then cut. In addition, the remaining nerves were destroyed by painting around the renal arteries with a solution of $20 \%$ phenol in ethanol. Finally, the arteries were washed with normal saline. The same procedures were performed on the MI group rats, except for damaging the nerves. The RDN procedure was conducted by the same investigators.

\section{Echocardiography}

Cardiac function was examined by echocardiography [11]. Echocardiography was performed in rats anesthetized with isoflurane at 2 weeks (before RDN) and repeated at 6 weeks after MI. A Vevo2100 (VisualSonics, Canada) system equipped with an MS-250, 16.0-21.0 MHz imaging transducer was applied for echocardiography. Heart rate (HR) was recorded at the same time. The investigators were blinded to the treatment of the rats. 


\section{Cellular Physiology Cell Physiol Biochem 2018;46:2471-2479 \begin{tabular}{c|c|c|} 
DOI: 10.1159/000489653 & O 2018 The Author(s). Published by S. Karger AG, Basel \\
www.karger.com/cpb
\end{tabular} \\ Zhang et al.: Renal Denervation on Ventricular Arrhythmias}

\section{Ventricular programmed electric stimulation}

Electrophysiological studies in vivo were performed at 4 weeks after RDN [12]. Electrocardiography was administered with 3 needle electrodes attached to the right upper limb and legs. Programmed electrical stimulation (PES) was used to stimulate the left ventricular apex of the heart through a bipolar electrode to investigate the incidence of VAs; the threshold potential for stable pacing was gained at a cycle length of $140 \mathrm{~ms}$. Pacing was started with twice as much as the threshold and a cycle length of $140 \mathrm{~ms}$, which was the interval of 8 stimuli (S1). An extra stimulus (S2) was applied until it failed to induce ventricular depolarization, while the interval (S1-S2) was shortened progressively by $10 \mathrm{~ms}$. The operators were blinded to the treatment of the rats.

\section{Immunofluorescence labeling}

Immunofluorescence labeling was used to investigate the distribution of connexin 43 (Cx43) in the infarcted border zone (IBZ) [13]. The specimens were evaluated under a fluorescence microscope (Nikon, Japan).

\section{Histopathological analysis}

As soon as each rat was sacrificed, the heart and kidneys were removed. The heart samples were cut transversely into 2 parts: one was fixed with $4 \%$ paraformaldehyde and then embedded in paraffin, while the other and the kidney samples were stored at $-80^{\circ} \mathrm{C}$. Sections cut from heart paraffin blocks were stained with Masson's trichrome to determine the degree of cardiac fibrosis with the collagen volume fraction (CVF), as described previously. At least 5 microscopic fields $(\times 100)$ were selected randomly to analyze CVF using Image-Pro Plus 6.0 software.

\section{Western blotting}

Western blotting was performed to identify the expression of transforming growth factor beta 1 (TGF- $\beta 1$ ), $\alpha$-smooth muscle actin ( $\alpha$-SMA), and Cx43 (Proteintech, China). Ventricle samples were lysed in lysis buffer (Beyotime, China), and the protein concentrations were determined using the BCA method, as described previously. Glyceraldehyde-3-phosphate dehydrogenase (Proteintech, China) was used to normalize protein levels.

\section{Enzyme-linked immunosorbent assay}

Plasma samples used for an enzyme-linked immunosorbent assay (ELISA) were obtained by centrifuging blood. Kidney samples were also homogenized and centrifuged to obtain supernatant. All samples were centrifuged at $3000 \mathrm{rpm}$. for $10 \mathrm{~min}$ at $4^{\circ} \mathrm{C}$. The serum levels of B-type natriuretic peptide (BNP) and aminoterminal pro-peptides of type I and III collagen (PINP and PIIINP, respectively) and the expression level of renal norepinephrine (NE) in the supernatant of kidney samples were tested using an ELISA kit (Elabscience Biotechnology, China). All procedures were performed according to the manufacturer's instructions

\section{Statistical analysis}

Continuous variables are presented as mean \pm standard deviation. For multiple comparisons, if the variance test was homogeneous, the data were analyzed by one-way analysis of variance followed by least significant difference test, or else Dunnett's 3T test was adopted. To compare the inducibility of VAs, Fisher's exact test was applied. SPSS 16.0 software (SPSS, Inc., USA) was used to analyze the data, and P values $<0.05$ were considered significant.

\section{Results}

Effectiveness of RDN

Renal NE levels were tested at 4 weeks after RDN in 3 groups (Fig. 1). Compared with the MI group, a significant decrease of NE was found in the MI+RDN group (MI+RDN 0.0213 $\pm 0.0034 \mathrm{ng} / \mathrm{mg}$ vs. MI $0.0324 \pm 0.0024 \mathrm{ng} / \mathrm{mg}, \mathrm{P}<0.001$ ), which proved the effectiveness of the RDN procedure. 
Cardiac function and HR at 2 weeks

At 2 weeks after MI, 8 rats in the control group and 17 rats treated with MI had survived. The 17 surviving MI rats were divided randomly into the MI group $(n=9)$ and MI+RDN group $(\mathrm{n}=8)$. Echocardiography was performed to measure cardiac structure and function. Left ventricular end systolic diameter (LVDs, all MI 7.142 $\pm 0.694 \mathrm{~mm}$ vs. control $5.126 \pm$ $0.267 \mathrm{~mm}, \mathrm{P}<0.001$ ) and left ventricular end diastolic diameter (LVDd, all MI $9.284 \pm 0.682$ $\mathrm{mm}$ vs. control $8.491 \pm 0.362 \mathrm{~mm}, \mathrm{P}<0.01$ ) were increased in the $17 \mathrm{MI}$ rats compared with the control rats (Fig. 2a-b and Table 1). Conversely, the ejection fraction (EF, all MI $44.33 \pm$ $4.75 \%$ vs. control $68.00 \pm 3.58 \%, \mathrm{P}<0.001$ ), fractional shortening (FS, all MI $23.15 \pm 2.94 \%$ vs. control $39.57 \pm 2.99 \%, \mathrm{P}<0.001$ ), interventricular septal thickness in systole (IVSs, all MI $1.795 \pm 0.515 \mathrm{~mm}$ vs. control $2.816 \pm 0.117 \mathrm{~mm}, \mathrm{P}<0.001)$, and interventricular septal thickness in diastole (IVSd, all MI $1.295 \pm 0.251 \mathrm{~mm}$ vs. control $1.736 \pm 0.072 \mathrm{~mm}, \mathrm{P}<0.001$ ) (Fig. 2c-f and Table 1) were decreased significantly in the 17 MI rats in comparison with the control rats, indicating the successful generation of the MI model. Furthermore, at this time, the rats that suffered MI had a higher HR than those that did not (all MI $363.5 \pm 33.9 \mathrm{bpm}$ vs. control $323.6 \pm 15.8 \mathrm{bpm}, \mathrm{P}<$ 0.01) (Fig. 2g).

Fig. 1. ELISA assay of renal norepinephrine. Renal norepinephrine level was significantly reduced through RDN compared with MI rats. $(\mathrm{n}=8,9$, 8 in Control, MI and MI+RDN group, respectively. Data were mean \pm SD. ${ }^{*} \mathrm{P}<0.05$ vs. Control group; \# $\mathrm{P}<0.05$ vs. MI group).

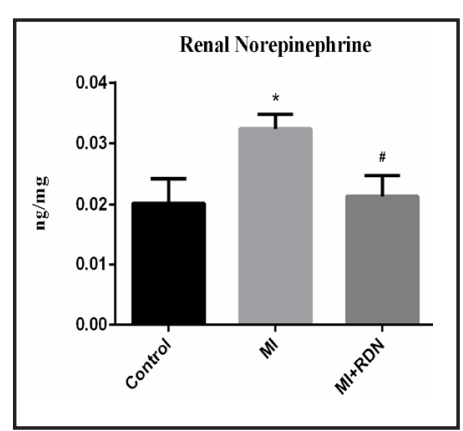

Table 1. Echocardiographic characteristics at Week 2 and $6 . \mathrm{EF}=$ ejection fraction; FS = fractional shortening; IVSd = interventricular septal thickness in diastole; IVSs = interventricular septal thickness in systole; LVDd= left ventricular end diastolic diameter; LVDs = left ventricular end systolic diameter; LAD = left atrial diameter

\begin{tabular}{lcccccccc}
\hline Group & Week & EF(100\%) & FS(100\%) & IVSd(mm) & IVSs(mm) & LVDd(mm) & LVDs(mm) & LAD(mm) \\
\hline Control & 2 & $68.00 \pm 3.58$ & $39.57 \pm 2.99$ & $1.74 \pm 0.07$ & $2.82 \pm 0.12$ & $8.49 \pm 0.36$ & $5.13 \pm 0.27$ & $4.87 \pm 0.45$ \\
& 6 & $67.37 \pm 4.81$ & $39.14 \pm 3.85$ & $1.75 \pm 0.06$ & $2.76 \pm 0.12$ & $8.56 \pm 0.34$ & $5.21 \pm 0.42$ & $5.05 \pm 0.49$ \\
MI & 2 & $44.30 \pm 4.87$ & $23.15 \pm 2.94$ & $1.21 \pm 0.29$ & $1.70 \pm 0.64$ & $9.31 \pm 0.80$ & $7.16 \pm 0.77$ & $4.85 \pm 0.70$ \\
& 6 & $43.40 \pm 7.52$ & $22.81 \pm 4.49$ & $1.18 \pm 0.39$ & $1.65 \pm 0.61$ & $10.24 \pm 1.10$ & $7.93 \pm 1.17$ & $4.92 \pm 0.99$ \\
MI+RDN & 2 & $44.36 \pm 4.94$ & $23.18 \pm 2.93$ & $1.39 \pm 0.17$ & $1.90 \pm 0.34$ & $9.25 \pm 0.57$ & $7.12 \pm 0.65$ & $4.87 \pm 0.39$ \\
& 6 & $56.91 \pm 4.44$ & $31.43 \pm 2.92$ & $1.71 \pm 0.47$ & $2.58 \pm 0.60$ & $9.44 \pm 1.04$ & $6.49 \pm 0.89$ & $4.53 \pm 0.35$ \\
\hline
\end{tabular}

Fig. 2. MI resulted in cardiac dysfunction at 2 weeks. Echocardiography evaluation of (a) LVDs (b) LVDd (c) EF (d) FS (e) IVSs (f) IVSd. The heart rate at 2 weeks (g) and 6 weeks (i). ELISA assay of plasma BNP at 6 weeks (h). ( $\mathrm{n}=8,9,8$ in Control, MI and MI+RDN group, Data were mean \pm SD. $* \mathrm{P}<0.05$ vs. Control group; \# $\mathrm{P}<0.05$ vs. MI group).LVDs = left ventricular end systolic diameter; LVDd= left ventricular end diastolic diameter; $\mathrm{EF}=$ ejection fraction; FS = fractional shortening; IVSs = interventricular septal thickness in systole; IVSd = interventricular septal thickness in diastole;

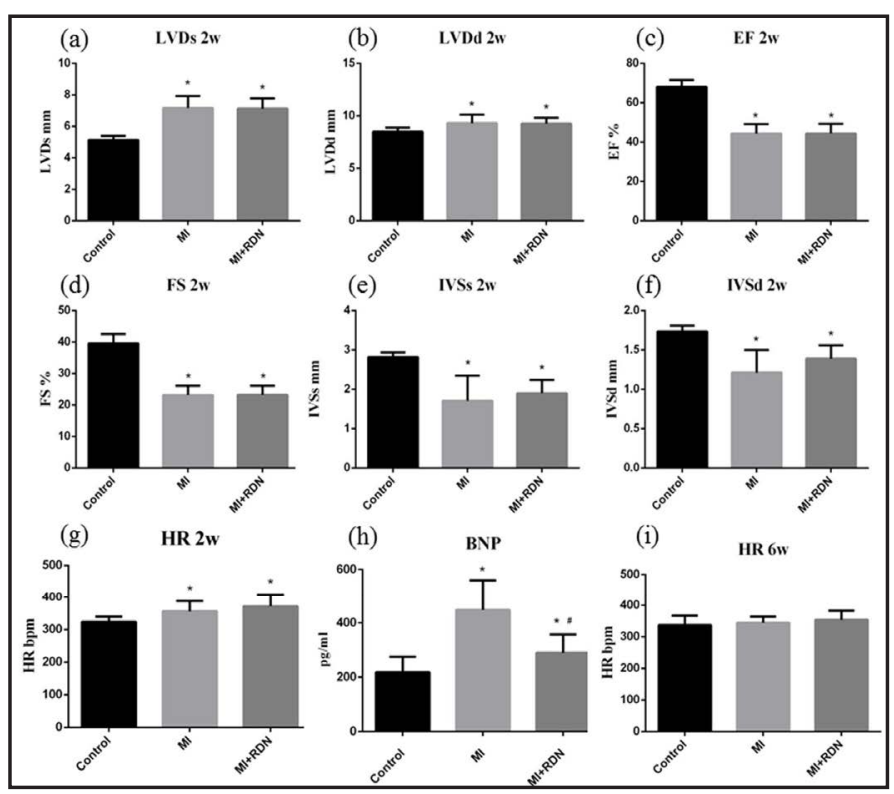


Cardiac function and HR at 6 weeks

At 6 weeks after MI, the changes in LVDs (MI+RDN $6.488 \pm 0.888 \mathrm{~mm}$ vs. MI $7.930 \pm$ $1.169 \mathrm{~mm}, \mathrm{P}<0.01$ ), LVDd (MI+RDN $9.441 \pm 1.043 \mathrm{~mm}$ vs. MI $10.240 \pm 1.097 \mathrm{~mm}, \mathrm{P}<0.05$ ), $\mathrm{EF}(\mathrm{MI}+\mathrm{RDN} 56.91 \pm 4.44 \%$ vs. MI $43.40 \pm 7.52 \%$, P < 0.001), FS (MI+RDN $31.43 \pm 2.92 \%$ vs. MI $22.81 \pm 4.49 \%, \mathrm{P}<0.001$ ), IVSs (MI+RDN $2.581 \pm 0.598 \mathrm{~mm}$ vs. MI $1.652 \pm 0.607 \mathrm{~mm}$, $\mathrm{P}<0.01$ ), and IVSd (MI+RDN $1.713 \pm 0.475 \mathrm{~mm}$ vs. MI $1.180 \pm 0.391 \mathrm{~mm}, \mathrm{P}<0.05$ ) were attenuated significantly in the MI+RDN group compared to the MI group (Fig. 3a-g and Table 1). In addition, there was no difference in LVDd (MI+RDN $9.441 \pm 1.043 \mathrm{~mm}$ vs. control 8.560 $\pm 0.339 \mathrm{~mm}, \mathrm{P}>0.05$ ), IVSs (MI+RDN $2.581 \pm 0.598 \mathrm{~mm}$ vs. control $2.756 \pm 0.119 \mathrm{~mm}, \mathrm{P}>$ $0.05)$, and IVSd (MI+RDN $1.713 \pm 0.475 \mathrm{~mm}$ vs. control $1.753 \pm 0.064 \mathrm{~mm}, \mathrm{P}>0.05$ ) between the MI+RDN group and the control group. In accordance with the echocardiography findings, the plasma BNP level was significantly higher in the MI group than in the MI+RDN group (MI+RDN $289.9 \pm 67.18 \mathrm{pg} / \mathrm{mL}$ vs. MI $447.8 \pm 112.3 \mathrm{pg} / \mathrm{mL}, \mathrm{P}<0.001$ ) (Fig. 2h). However, no difference was found in HR among the 3 groups at this time point (control $336.9 \pm 30.6 \mathrm{bpm}$ vs. MI+RDN $353.5 \pm 29.8$ bpm vs. MI $344.2 \pm 19.6$ bpm, P > 0.05) (Fig. 2i).

\section{Effects of RDN on VAs}

The outcomes of ventricular PES are shown in Fig. 4a-c. VAs, including ventricular tachycardia and ventricular fibrillation, were induced more easily in the MI group than in the MI+RDN group. In detail, induced VAs were present in 8/9 rats from the MI group and 3/8 rats from the MI+RDN group $(\mathrm{P}<0.05)$, while only $1 / 8$ rats from the control group suffered such consequences (Fig. 4 d).

\section{Effects of RDN on fibrosis}

CVF was used to estimate the degree of cardiac fibrosis through Masson's staining. Obvious fibrosis was observed in the MI-treated rats compared with the control rats, while the RDN procedure significantly attenuated CVF (MI+RDN $31.10 \pm 3.97 \%$ vs. MI $54.80 \pm$ $16.39 \%, \mathrm{P}<0.001$ ) (Fig. 5). The expression of TGF- $\beta 1$ and $\alpha$-SMA, both of which are fibrosisrelated factors, was significantly up-regulated in the heart by coronary occlusion. However, in the MI+RDN group, these changes were partially repaired (Fig. 6a). In accordance with this, the plasma levels of P I NP (MI+RDN $41.44 \pm 10.10 \mathrm{ng} / \mathrm{mL}$ vs. MI $95.49 \pm 24.83 \mathrm{ng} / \mathrm{mL}$, $\mathrm{P}<0.001$ ) and PIIINP (MI+RDN $82.12 \pm 30.79 \mathrm{ng} / \mathrm{mL}$ vs. MI $124.60 \pm 26.64 \mathrm{ng} / \mathrm{mL}, \mathrm{P}<0.05$ ) were significantly decreased in the MI+RDN group compared with the MI group (Fig. 6b).

Fig. 3. RDN partly restored cardiac dysfunction at 6 weeks. Echocardiography assessment of (a) LVDs (b) LVDd (c) EF (d) FS (e) IVSs (f) IVSd. Representative tracings of echocardiography (g). ( $\mathrm{n}=8,9,8$ in Control, MI and MI+RDN group, Data were mean \pm SD. ${ }^{*} \mathrm{P}<0.05$ vs. Control group; \# $\mathrm{P}<0.05$ vs. $\mathrm{MI}$ group).

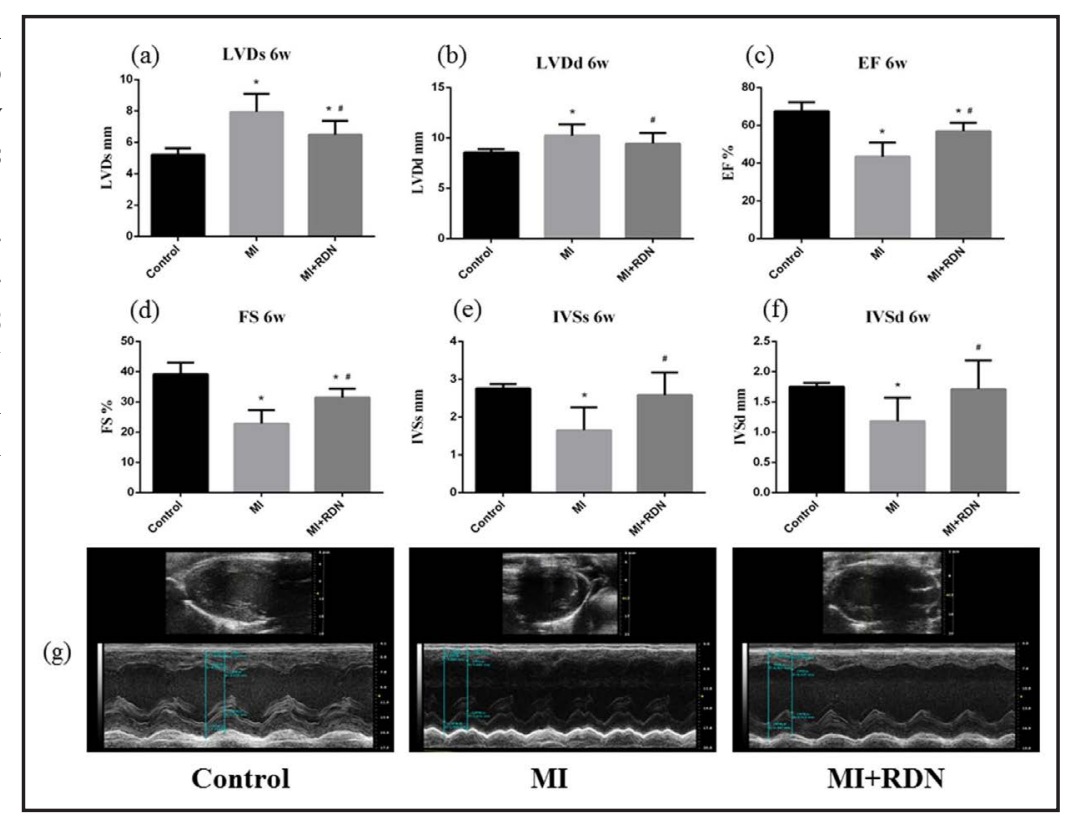


Fig. 4. RDN significantly reduced the inducibility of ventricular arrhythmias.Representative ECG of electrical stimulation, including sinus rhythm (a), ventricular tachycardia (b) and ven-

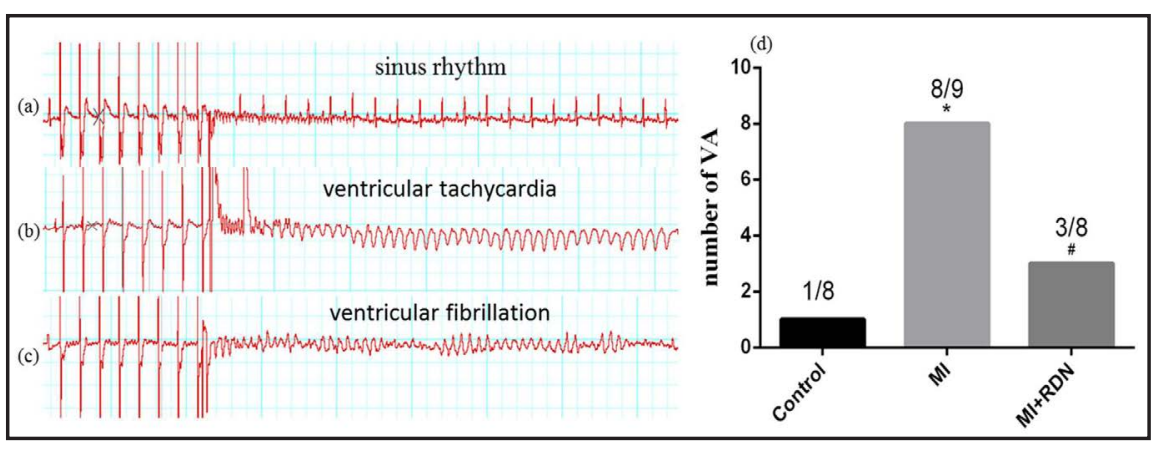
tricular fibrillation

(c). Ventricular arrhythmias were more easily induced in MI group rather than in MI+RDN group (d). ( $n=8$, 9, 8 in Control, $\mathrm{MI}$ and $\mathrm{MI}+\mathrm{RDN}$ group, ${ }^{*} \mathrm{P}<0.05$ vs. Control group; \# $\mathrm{P}<0.05$ vs. MI group).

Fig. 5. RDN significantly attenuated cardiac fibrosis. Representative ventricular fibrosis (in blue) by Masson's staining of samples from the control, MI, and MI+RDN groups. Quantitative analysis suggested that CVF in MI+RDN group was significantly lower than that in MI group. ( $\mathrm{n}=8$, 9, 8 in Control,

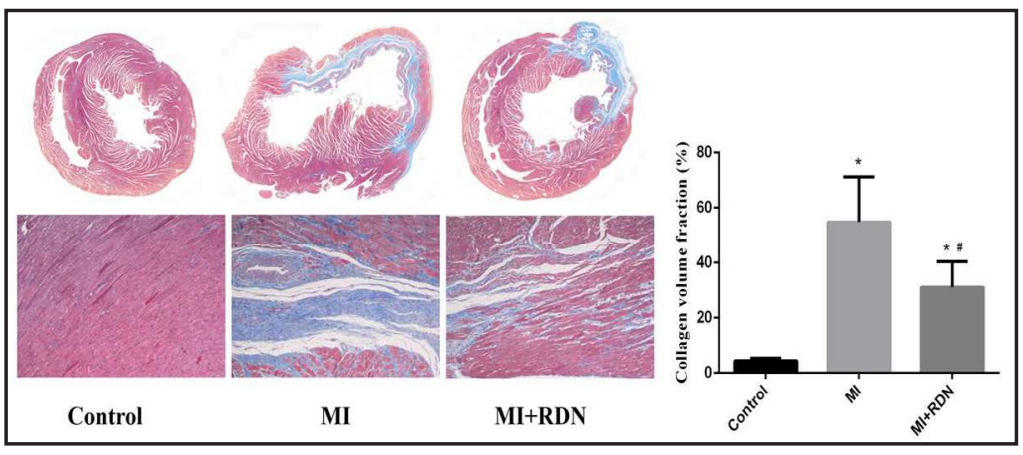
$\mathrm{MI}$ and MI+RDN group, Data were mean $\pm \mathrm{SD}$. ${ }^{*} \mathrm{P}<0.05$ vs. Control group; $\# \mathrm{P}<0.05$ vs. MI group).

Fig. 6. RDN lowered the expression of TGF- $\beta 1, \alpha-S M A$, P I NP and PIIINP.Quantitative analysis and representative protein expression of TGF- $\beta 1$ and $\alpha$-SMA in heart by Western blot (a). Quantitative analysis of the plasma levels of P I NP and PIIINP by Elisa assay (b). (n $=8,9,8$ in Control, MI and $\mathrm{MI}+\mathrm{RDN}$ group, Data were mean $\pm \mathrm{SD}$. ${ }^{*} \mathrm{P}<0.05$ vs. Control group; \# $\mathrm{P}<0.05$ vs. MI group).

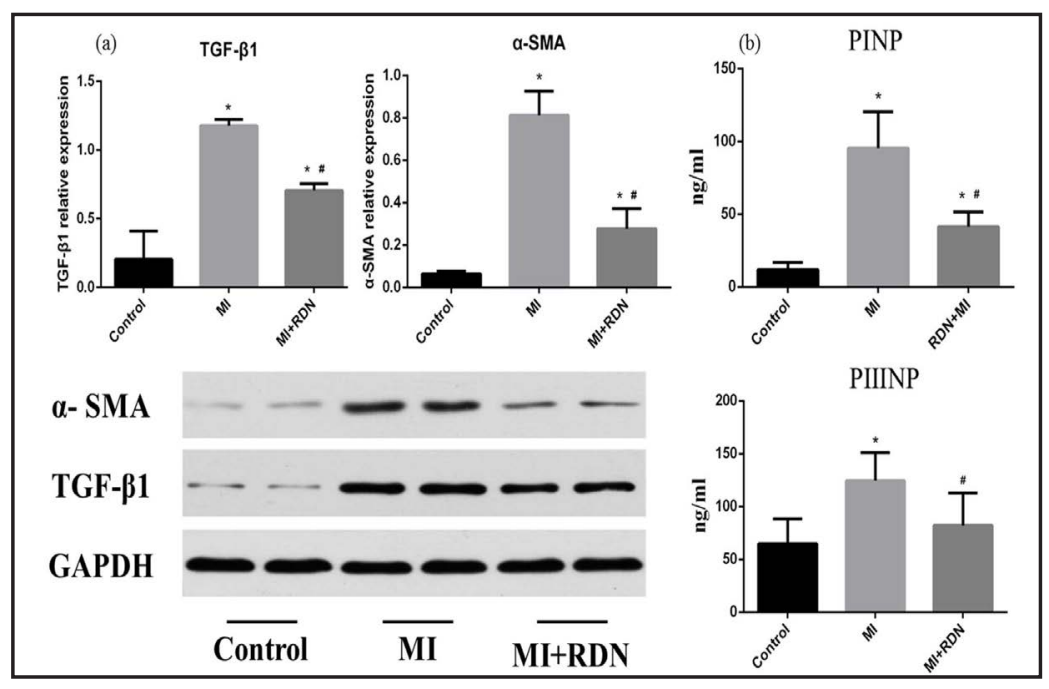

\section{Effects of RDN on Cx43}

Immunofluorescence analysis was chosen to evaluate the localization of $\mathrm{Cx} 43$. In the IBZ, the distribution of $\mathrm{Cx} 43$ was disrupted. This disruption of $\mathrm{Cx} 43$ localization was attenuated in the IBZ by the RDN procedure (Fig. 7a). The protein level of $\mathrm{Cx} 43$ was examined through western blotting. Compared with the control group, the relative expression of $\mathrm{Cx} 43$ was significantly reduced in the MI group; however, this decrease was reversed by RDN $(\mathrm{P}<0.01)$ (Fig. 7b).

\section{KARGER}


Fig. 7. Distribution and expression of $\mathrm{Cx} 43$ in the border-zone of the infarcted area from the control, MI, and MI+RDN groups.The distribution of $\mathrm{Cx} 43$ was disrupted in the border-zone of the infarcted area and the chaos of $\mathrm{Cx} 43$ was attenuated by RDN (a). Quantitative analysis and representative protein expression of $\mathrm{Cx} 43$

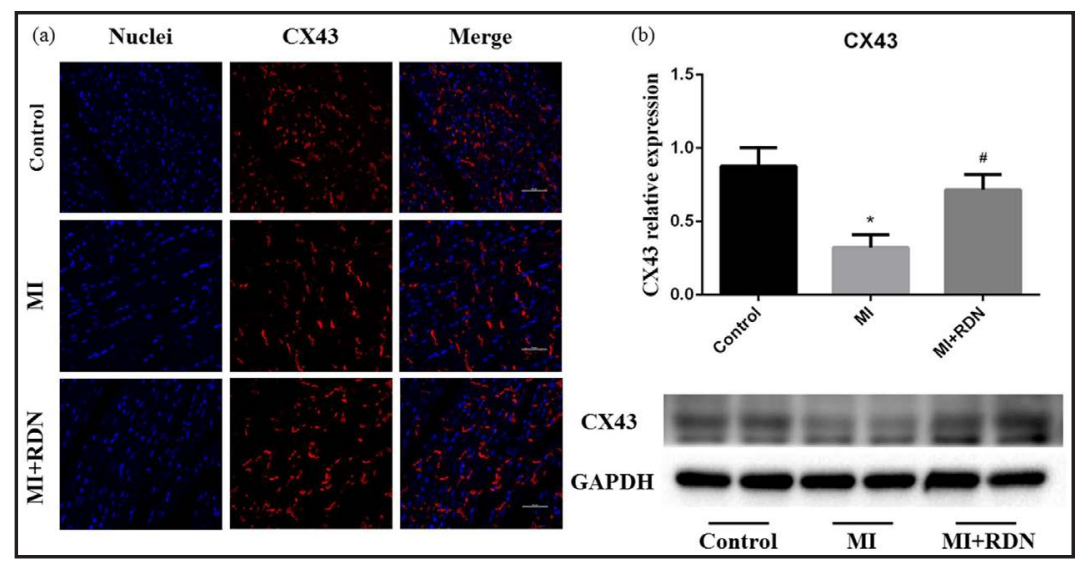
in heart by Western blot (b). ( $\mathrm{n}=8,9,8$ in Control, $\mathrm{MI}$ and $\mathrm{MI}+\mathrm{RDN}$ group, Data were mean $\pm \mathrm{SD}$. ${ }^{*} \mathrm{P}<0.05$ vs. Control group; \# $\mathrm{P}<0.05$ vs. MI group).

\section{Discussion}

The main findings of this study are as follows: (1) RDN attenuates VA inducibility in ischemic cardiomyopathy; (2) RDN significantly reduces myocardial fibrosis in ischemic cardiomyopathy; (3) RDN abolishes the abnormal distribution and quantity of $\mathrm{Cx} 43$; and (4) RDN has protective effects against cardiac dysfunction in MI rats.

Malignant VAs accompanied with severe hemodynamic disturbances are the major cause of sudden cardiac death after MI $[1,14]$. Medical treatment and an implantable cardioverter defibrillator (ICD) are the most commonly used therapeutic approaches for VAs. However, the development of anti-arrhythmic drugs alone is not sufficient, while the implantation of a cardioverter defibrillator is very expensive and has several side effects [15]. Thus, new techniques should be explored for the treatment of VAs.

Cardiac dysfunction is one of the predictors of VAs in ischemic cardiomyopathy [16]. In the present study, left ventricular dilation, compensatory myocardial hypertrophy, and poor cardiac function occurred in rats that suffered a coronary occlusion at 2 weeks after MI. However, impaired heart function was partially restored through the RDN procedure. In addition, the plasma levels of BNP, which is mainly released from the ventricles and is associated with a higher mortality in heart failure [17], were significantly decreased in MI rats with RDN compared with the MI group. These observations were similar to the findings of $\mathrm{Hu}$ et al. $[18,19]$.

Previous studies have investigated the influences of RDN on VAs in ischemic heart disease. Huang et al. demonstrated that RDN can decrease the incidence of VAs in an experimental model of acute myocardial infarction in dogs [20]. Similarly, Linz et al. reported that RDN suppressed VAs during acute ischemic events in pigs [6]. However, only a few studies have focused on the role of RDN against VAs in a model of MI-induced ischemic cardiomyopathy. Nicholas et al. recently showed that RDN can reduce spontaneous VAs after MI, and this phenomenon may be associated with the downregulation of cardiac sympathetic activity [7]. In the present study, RDN indeed decreased the occurrence of VAs in ischemic heart disease, which was consistent with the findings of Jacksonet al. [7].

Fibrosis is a common pathway leading to organ injury and failure [21]. In response to variable environment stimuli, including myocardial infarction, structural remodeling occurs in the heart, with a massive deposition of extracellular matrix. These pathological changes may increase the risks of heart failure and malignant arrhythmias [22, 23]. In fact, a study showed that a $3 \%$ increase in extracellular volume fraction contributes to a $50 \%$ increase in cardiovascular events [24]. In our study, the protein expression levels of TGF- $\beta 1$ and $\alpha$-SMA, which are common fibrosis-related factors, were significantly downregulated, and 
the plasma levels of PINP and PIIINP, known as specific biomarkers involved in collagen type I and III synthesis [25], were decreased in the MI+RDN group compared with the MI group. Thus, the RDN procedure indeed reduced myocardial fibrosis in ischemic hearts and could reduce the frequency of VAs.

Numerous gap junctions located between cardiomyocytes facilitate conduction through normal ventricular muscle in a continuous process [26]. However, there are fewer and smaller gap junctions between myocytes located near infarcts [27] Thus, conduction velocity is reduced. Actually, conditions that decrease conduction velocity also promote reentry, while reentry plays a vital role in the formation of VAs in infarcted hearts [26]. In the present study, the abnormal distribution and quantity of $\mathrm{Cx} 43$ were attenuated by the RDN procedure, which may help to improve conduction velocity and reduce reentry. Therefore, the effects of RDN on antiarrhythmic were probably related to the preservation of $\mathrm{Cx} 43$ in infarcted hearts.

Several limitations of this study should be noted. First, the major etiology of clinical MI is coronary atherosclerosis, while we induced MI by ligating the left anterior descending coronary artery. As a result, the pathophysiological state of the experimental ischemic cardiomyopathy model may be different from the actual state. Second, epicardial monophasic action potentials were not recorded during PES, so we could not calculate action potential duration at $90 \%$ repolarization or the dispersion of action potential duration, which are both increased in post-infarcted hearts [12].

\section{Conclusion}

RDN reduced myocardial fibrosis and suppressed VAs in a rat model of ischemic cardiomyopathy.

\section{Acknowledgements}

This work was supported by grants from the National Natural Science Foundation of China (No 81770333), the National Natural Science Youth Foundation of China (NO 81400253), the Natural Science Youth Foundation of the Jiangsu Province of China (No. BK20141029), the Natural Science Fund of Health Administration of Jiangsu Province (No. H201302), the Scientific Research Program for 333 Project of Jiangsu Province (No. BRA2017190), and the Priority Academic Program Development of Jiangsu Higher Education Institutions.

\section{Disclosure Statement}

The authors declare that no conflict of interests exists.

\section{References}

1 Bunch TJ, Hohnloser SH, Gersh BJ: Mechanisms of sudden cardiac death in myocardial infarction survivors: insights from the randomized trials of implantable cardioverter-defibrillators. Circulation 2007;115:24512457.

-2 Symplicity HTNI, Esler MD, Krum H, Sobotka PA, Schlaich MP, Schmieder RE, Bohm M: Renal sympathetic denervation in patients with treatment-resistant hypertension (The Symplicity HTN-2 Trial): a randomised controlled trial. Lancet 2010;376:1903-1909.

-3 Wang K, Lu D, Zhang B, Wang S, Liu Q, Zhang Q, Geng J, Shan Q: Renal Denervation Attenuates MultiOrgan Fibrosis and Improves Vascular Remodeling in Rats with Transverse Aortic Constriction Induced Cardiomyopathy. Cell Physiol Biochem 2016;40:465-476.

-4 Liu Q, Zhang Q, Wang K, Wang S, Lu D, Li Z, Geng J, Fang P, Wang Y, Shan Q: Renal Denervation Findings on Cardiac and Renal Fibrosis in Rats with Isoproterenol Induced Cardiomyopathy. Sci Rep 2015;5:18582.

5 Mahfoud F, Urban D, Teller D, Linz D, Stawowy P, Hassel JH, Fries P, Dreysse S, Wellnhofer E, Schneider G, Buecker A, Schneeweis C, Doltra A, Schlaich MP, Esler MD, Fleck E, Bohm M, Kelle S: Effect of renal 


\section{Cellular Physiology Cell Physiol Biochem 2018;46:2471-2479 \begin{tabular}{lll} 
DOI: 10.1159/000489653 & and Biochemistry & $\begin{array}{l}\text { O } 2018 \text { The Author(s). Published by S. Karger AG, Basel } \\
\text { www.karger.com/cpb }\end{array}$ \\
\cline { 2 - 3 }
\end{tabular}

denervation on left ventricular mass and function in patients with resistant hypertension: data from a multi-centre cardiovascular magnetic resonance imaging trial. Eur Heart J 2014;35:2224-2231b.

-6 Linz D, Wirth K, Ukena C, Mahfoud F, Poss J, Linz B, Bohm M, Neuberger HR: Renal denervation suppresses ventricular arrhythmias during acute ventricular ischemia in pigs. Heart Rhythm 2013;10:1525-1530.

-7 Jackson N, Gizurarson S, Azam MA, King B, Ramadeen A, Zamiri N, Porta-Sanchez A, Al-Hesayen A, Graham J, Kusha M, Masse S, Lai PF, Parker J, John R, Kiehl TR, Nair GK, Dorian P, Nanthakumar K: Effects of Renal Artery Denervation on Ventricular Arrhythmias in a Postinfarct Model. Circ Cardiovasc Interv 2017;10:e004172.

8 Luo Q Guo D, Liu G, Chen G, Hang M, Jin M: Exosomes from MiR-126-Overexpressing Adscs Are Therapeutic in Relieving Acute Myocardial Ischaemic Injury. Cell Physiol Biochem 2017;44:2105-2116.

-9 Xiao J, Yu K, Li M, Xiong C, Wei Y, Zeng Q: The IL-2/Anti-IL-2 Complex Attenuates Cardiac IschaemiaReperfusion Injury Through Expansion of Regulatory T Cells. Cell Physiol Biochem 2017;44:1810-1827.

10 Lu D, Wang K, Wang S, Zhang B, Liu Q Zhang Q, Geng J, Shan Q: Beneficial effects of renal denervation on cardiac angiogenesis in rats with prolonged pressure overload. Acta Physiol (Oxf) 2017;220:47-57.

11 Tong G, Zhang B, Zhou X, Zhao J, Sun Z, Tao Y, Pei J, Zhang W: Kappa-Opioid Agonist U50, 488H-Mediated Protection Against Heart Failure Following Myocardial Ischemia/Reperfusion: Dual Roles of Heme Oxygenase-1 Cell Physiol Biochem 2016;39:2158-2172.

12 Wang D, Zhu H, Yang Q Sun Y: Effects of relaxin on cardiac fibrosis, apoptosis, and tachyarrhythmia in rats with myocardial infarction. Biomed Pharmacother 2016;84:348-355.

13 Gu Y, Wu G, Wang X, Wang X, Wang Y, Huang C: Artemisinin prevents electric remodeling following myocardial infarction possibly by upregulating the expression of connexin 43. Mol Med Rep 2014;10:18511856.

14 Schomig A: Catecholamines in myocardial ischemia. Systemic and cardiac release. Circulation 1990;82:II1322.

-15 Godemann F, Butter C, Lampe F, Linden M, Schlegl M, Schultheiss HP, Behrens S: Panic disorders and agoraphobia: side effects of treatment with an implantable cardioverter/defibrillator. Clin Cardiol 2004;27:321-326.

16 Naccarella F, Lepera G, Rolli A: Arrhythmic risk stratification of post-myocardial infarction patients. Curr Opin Cardiol 2000;15:1-6.

-17 Scirica BM, Kadakia MB, de Lemos JA, Roe MT, Morrow DA, Li S, Wiviott SD, Kontos MC, National Cardiovascular Data R: Association between natriuretic peptides and mortality among patients admitted with myocardial infarction: a report from the ACTION Registry(R)-GWTG. Clin Chem 2013;59:1205-1214.

-18 Hu J, Ji M, Niu C, Aini A, Zhou Q Zhang L, Jiang T, Yan Y, Hou Y: Effects of renal sympathetic denervation on post-myocardial infarction cardiac remodeling in rats. PLoS One 2012; 7:e45986.

19 Hu J, Li Y, Cheng W, Yang Z, Wang F, Lv P, Niu C, Hou Y, Yan Y, Ge J: A comparison of the efficacy of surgical renal denervation and pharmacologic therapies in post-myocardial infarction heart failure. PLoS One 2014;9:e96996.

20 Huang B, Yu L, He B, Lu Z, Wang S, He W, Yang K, Liao K, Zhang L, Jiang H: Renal sympathetic denervation modulates ventricular electrophysiology and has a protective effect on ischaemia-induced ventricular arrhythmia. Exp Physiol 2014;99:1467-1477.

21 Rockey DC, Bell PD, Hill JA: Fibrosis--A Common Pathway to Organ Injury and Failure. N Engl J Med 2015;373:96.

22 Hill JA, Olson EN: Cardiac plasticity. N Engl J Med 2008;358:1370-1380.

-23 Spinale FG: Myocardial matrix remodeling and the matrix metalloproteinases: influence on cardiac form and function. Physiol Rev 2007;87:1285-1342.

-24 Wong TC, Piehler K, Meier CG, Testa SM, Klock AM, Aneizi AA, Shakesprere J, Kellman P, Shroff SG, Schwartzman DS, Mulukutla SR, Simon MA, Schelbert EB: Association between extracellular matrix expansion quantified by cardiovascular magnetic resonance and short-term mortality. Circulation 2012;126:1206-1216.

-25 Lepojarvi ES, Piira OP, Paakko E, Lammentausta E, Risteli J, Miettinen JA, Perkiomaki JS, Huikuri HV, Junttila MJ: Serum PINP, PIIINP, galectin-3, and ST2 as surrogates of myocardial fibrosis and echocardiographic left venticular diastolic filling properties. Front Physiol 2015;6:200.

26 Saffitz JE: The pathology of sudden cardiac death in patients with ischemic heart disease--arrhythmology for anatomic pathologists. Cardiovasc Pathol 2005;14:195-203.

-27 Luke RA, Saffitz JE: Remodeling of ventricular conduction pathways in healed canine infarct border zones. J Clin Invest 1991;87:1594-1602. 